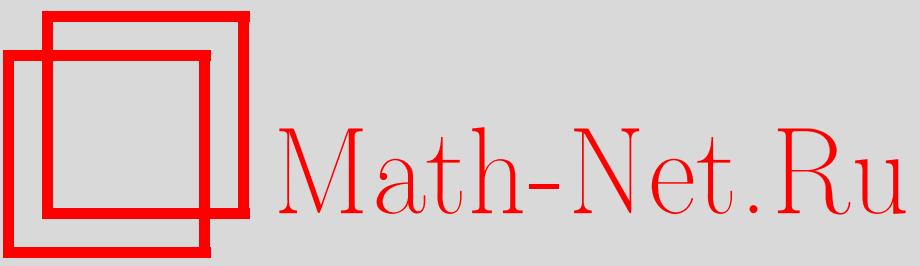

В. Л. Боднева, А. А. Лундин, А. А. Милютин, О возможности сокращенного описания спиновой динамики анизотропных гейзенберговских парамагнетиков и форме спектров ЯМР в твердом теле, ТМФ, 1996, том 106, номер 3, 452-468

DOI: https://doi.org/10.4213/tmf1129

Использование Общероссийского математического портала Math-Net.Ru подразумевает, что вы прочитали и согласны с пользовательским соглашением

http://www.mathnet.ru/rus/agreement

Параметры загрузки:

IP : 3.85 .5 .30

26 апреля 2023 г., 17:46:09 
ТЕОРЕТИЧЕСКАЯ

И МАТЕМАТИЧЕСКАЯ

ФИЗИКА

Том 106, № 3

март, 1996

\author{
В. Л. Боднева, А. А. Лундин, А. А. Милютин
}

\title{
О ВОЗМОЖНОСТИ СОКРАШЕННОГО ОПИСАНИЯ СПИНОВОЙ ДИНАМИКИ АНИЗОТРОПНЫХ ГЕЙЗЕНБЕРГОВСКИХ ПАРАМАГНЕТИКОВ И ФОРМЕ СПЕКТРОВ ЯМР В ТВЕРДОМ ТЕЛЕ
}

\footnotetext{
Задача о форме линии ЯМР в твердом теле решена в квадратурах. Хотя построенное решение опирается на полученные в работе точные результаты, возможность корректного описания экспериментальных данных на основе низших приближений теории сушественно зависит от типа кристаллической решетки: в низших приближениях адекватно описываются только спектры кристаллов с обычными плотными решетками, что, впрочем, и является наиболее актуальной задачей (а не квазиодномерные системы, например). Результаты теории сравниваются с экспериментальными. Получено хорошее согласие между теорией и экспериментом.
}

\section{1. ВВЕДЕНИЕ}

Несмотря на то что теоретическим и экспериментальным исследованиям динамики парамагнитных многоспиновых систем посвяшены сотни работ (см., например, [1-6] и библиографию в этих статьях), развитие этой области статистической механики далеко не завершено и исследования не имеют тенденции к сокращению.

Термин "спиновая динамика" подразумевает совокупность процессов, протекающих в спиновых системах под влиянием внутренних спин-спиновых взаимодействий в условиях изоляции от остальных степеней свободы образца (решетки).

Под влиянием этих внутренних взаимодействий изначально неравновесная (в силу каких-либо внешних причин) спиновая система приходит в состояние частичного или полного (в зависимости от условий и времени наблюдения) внутреннего равновесия.

Интерес к этой проблематике обусловлен как ее фундаментальной значимостью для описания экспериментов по магнитному резонансу по наблюдению магнитной части рассеяния нейтронов и пр., так и той сушественной ролью, которую развитие спиновой динамики могло бы сыграть для понимания физики необратимых процессов.

Применительно к ЯМР основная задача спиновой динамики состоит в вычислении временной корреляционной функции (BKФ), описывающей релаксацию поперечной по отношению к внешнему магнитному полю компоненты намагниченности (сигнал свободной прецессии (ССП) - фурье-образ линии поглощения). Проблема выгисления формы линии ЯМР в твердом теле имеет обширную предысторию. Первые предположения о форме линии были высказаны в пионерской работе Бломбергена, Перселла и Паунда [7]. Вначале предполагалось [7-10], что линии ЯМР должны иметь лоренцеву либо гауссову форму. При этом лоренцевыми линиями должны описываться спектры в жидкостях 
или твердых телах с сильным обменным взаимодействием между спинами, т.е. спектры, возникающие при наличии быстрых флуктуаций локальных магнитных полей, создаваемых окружением на некотором спине. В принципе уширение резонансных линий в твердых телах может обусловливаться целым рядом физических причин. Однако очень часто определяющим взаимодействием, которое вызывает уширение, является так называемая секулярная часть диполь-дипольного взаимодействия [8] между магнитными моментами ядер. В этом случае гауссовым линиям должно соответствовать распределение дипольных магнитных полей статического типа, которое, как предполагалось, и реализуется в большинстве твердых тел.

Однако еще в 1957 г. Лоу и Норберг [11] экспериментально показали, что ССП монокристалла $\mathrm{CaF}_{2}$ - классического объекта для исследования спиновой динамики в ЯМР содержит осцилляции, и, таким образом, форма линии не является гауссовой. С тех пор для решения этой задачи было предложено множество различных подходов, причем часто весьма общего характера, позволяющих в той или иной степени добиться согласия с экспериментом. Однако практически все работы используют интуитивные феноменологические предположения. Критический обзор предшествуюших работ можно найти в статьях $[4,6,12]$. И хотя основной причиной, препятствовавшей до сих пор успешному разрешению проблемы, является многочастичность задачи при отсутствии в гамильтониане малого параметра, в настоящей работе представлена теория, опирающаяся на некоторые полученные в работе точные результаты.

\section{2. ОСНОВНЫЕ УРАВНЕНИЯ СПИНОВОЙ ДИНАМИКИ}

Гамильтониан спиновой системы с анизотропным гейзенберговским взаимодействием имеет вид

$$
H=\sum_{i>j}\left\{\Delta_{i j} S_{z_{i}} S_{z_{j}}+I_{i j}\left(S_{i}^{+} S_{j}^{-}+S_{i}^{-} S_{j}^{+}\right)\right\}
$$

при $\Delta_{i j}=b_{i j} ; I_{i j}=-\frac{1}{4} b_{i j}, b_{i j}=\frac{\gamma^{2} \hbar^{2}}{r_{i j}^{3}}\left(1-3 \cos ^{2} \vartheta_{i j}\right)$.

Выражение (1) описьвает секулярную часть диполь-дипольного взаимодействия, ответственного за уширение линии ЯМР в твердом теле [8]. Здесь $r_{i j}$-расстояние между спинами $i$ и $j$ в решетке, $\vartheta_{i j}$ - угол между направлением зеемановского поля $H_{0}$ и вектором $\vec{r}_{i j}, \gamma$ - гиромагнитное отношение ядер. В системе координат, врашающейся с ларморовской частотой $\gamma H_{0}$, наблюдаемый ССП пропорционален временной корреляционной функции [8]:

$$
A_{0}(t)=\frac{1}{\operatorname{Sp}\left\{S_{x}^{2}\right\}}\left\{S_{x}(t) S_{x}\right\}
$$

$S_{x}=\sum_{i} S_{x_{i}}-x$-компонента суммарного спина системы, $S_{x}(t)$ - решение гейзенберговского уравнения

$$
\dot{S}_{x}=\frac{1}{\hbar}\left[H, S_{x}\right]
$$

Формула (2) - следствие высокотемпературного приближения, с огромной точностью справедливого для ЯМР [8].

В работе [13] было показано, что задача о вычислении ВКФ (2) полностью эквивалентна решению бесконечной (размерность $10^{23}$ ) системы дифференциальных уравне- 
ний

$$
\begin{aligned}
& \dot{A}_{0}=i \nu_{0}^{2} A_{1}, \\
& \dot{A}_{1}=i\left(A_{0}+\nu_{1}^{2} A_{2}\right), \\
& \ldots \ldots \ldots \ldots \ldots \ldots \ldots \\
& \dot{A}_{n}=i\left(A_{n-1}+\nu_{n}^{2} A_{n+1}\right), \\
& \ldots \ldots \ldots \ldots \ldots \ldots \ldots
\end{aligned}
$$

с начальными условиями $A_{0}(0)=1, A_{n}(0)=0, n \geqslant 1$. Функции $\left\{A_{i}(t)\right\}$ - "многокоммутаторные" (многочастичные) ВКФ, поскольку каждое дифференцирование ССП (2), добавляя операцию комму тирования, добавляет тем самым еше один оператор с новым, вообще говоря, индексом суммирования по решетке. Параметры $\left\{\nu_{i}^{2}\right\}$, свойства которых и определяют решение системы, связаны с моментами линии поглощения

$$
\nu_{n}^{2}=D_{n-1} D_{n+1} / D_{n}^{2},
$$

$\left\{D_{n}\right\}$ - определители, имеюшие вид

$$
D_{n}=\left|\begin{array}{cccc}
1 & M_{1} & \ldots & M_{n} \\
M_{1} & M_{2} & \ldots & M_{n+1} \\
\ldots \ldots & \ldots & \ldots & \ldots . \\
M_{n} & M_{n+1} & \ldots & M_{2 n}
\end{array}\right|,
$$

здесь $\left\{M_{j}\right\}$ - моменты, коэффициенты разложения в ряд по степеням времени ССП:

$$
A_{0}(t)=\sum_{n=0}^{\infty} i^{2 n} \frac{M_{2 n}}{2 n !} t^{2 n} .
$$

Вследствие парамагнетизма системы (высокие температуры) лиш моменты четного порядка в (6), (7) отличны от нуля. Для удобства приведем выражения нескольких первых коэффициентов:

$$
\begin{aligned}
D_{-1} & =D_{0}=1, \quad D_{1}=M_{2}, \quad D_{2}=M_{2}\left(M_{4}-M_{2}^{2}\right), \\
D_{3} & =\left(M_{4}-M_{2}^{2}\right)\left(M_{2} M_{6}-M_{4}^{2}\right), \\
\nu_{0}^{2} & =M_{2}, \quad \nu_{1}^{2}=\frac{M_{4}-M_{2}^{2}}{M_{2}}, \quad \nu_{2}^{2}=\frac{M_{2} M_{6}-M_{4}^{2}}{M_{2}\left(M_{4}-M_{2}^{2}\right)} .
\end{aligned}
$$

Соотношения (4)-(8), определенные в принципе для всего кристалла, можно, поскольку поведение решения системы полностью определяется коэффициентами $\left\{\nu_{n}^{2}\right\}$, отнести только к компоненте ССП, обусловленной спинами ячейки $[14,3]$. В работах $[14,3]$ показано, что в кристаллах с большим числом ближайших соседей, окружаюших некоторый выделенный (любой) спин решетки, весь кристалл можно разбить по отношению к выделенному спину на две области. В одной из них спины движутся коррелированно по отношению к выделенному спину, а в другой - нет. Область с коррелированным движением была названа ячейкой $[14,3]$. Подчеркнем, что в силу глубочайшего парамагнетизма системы пространственные корреляции полностью отсутствуют и речь идет исключительно о временных корреляциях, возникаюших благодаря слагаемому $H_{f f}$ в гамильтониане (1). В то время как компонента ССП, обусловленная спинами, расположенными вне ячейки, может быть описана достаточно просто $[14,3]$, расчет вклада в 
ССП от ячейки весьма нетривиален именно вследствие существенной роли корреляционных эффектов.

Таким образом, хотя абсолютное большинство формальных результатов, полученных ниже, справедливо вне зависимости от каких бы то ни было модельных предположений, конкретные расчеты, необходимые для сопоставления теории и эксперимента, проведены в рамках модели ячейки $[14,3]$. Дополнительное обсуждение некоторых аспектов выделения ячейки проведено в приложении 1.

\section{3. СИММЕТРИЗАЦИЯ ОСНОВНОЙ СИСТЕМЫ УРАВНЕНИЙ}

Для последуюшего решения систему уравнений (4) целесообразно симметризовать. Для этого сделаем преобразование

$$
A_{0}=B_{0}, \quad A_{k}=s_{k} B_{k} \quad(k=1,2, \ldots) .
$$

Получим систему

$$
\begin{aligned}
& \dot{\widetilde{B}}_{0}=i \nu_{0}^{2} s_{1} B_{1}, \\
& \dot{\vec{B}}_{1}=i \frac{1}{s_{1}}\left(\widetilde{B}_{0}+\nu_{1}^{2} s_{2} \widetilde{B}_{2}\right), \\
& \dot{\widetilde{B}}_{2}=i \frac{1}{s_{2}}\left(s_{1} \widetilde{B}_{1}+\nu_{2}^{2} s_{3} \widetilde{B}_{3}\right), \\
& \dot{\widetilde{B}}_{n}=i \frac{1}{s_{n}}\left(s_{n-1} \widetilde{B}_{n-1}+\nu_{n}^{2} s_{n+1} \widetilde{B}_{n+1}\right), \\
& \ldots \ldots \ldots \ldots \ldots \ldots \ldots \ldots \ldots
\end{aligned}
$$

При заданном $s_{1}$ остальные $\left\{s_{k}\right\}$ выбираются для симметризации системы однозначно:

$$
\begin{gathered}
\nu_{1}^{2} s_{2}=1 \Rightarrow s_{2}=\frac{1}{\nu_{1}^{2}}, \\
\nu_{2}^{2} s_{3}=1 \Rightarrow s_{3}=\frac{s_{1}}{\nu_{2}^{2}}, \\
\ldots \ldots \ldots \ldots \ldots \ldots \ldots \ldots, \ldots \ldots \ldots, \\
\nu_{n}^{2} s_{n+1}=s_{n-1} \Rightarrow s_{n+1}=\frac{s_{n-1}}{\nu_{n}^{2}} .
\end{gathered}
$$

В то же время, если система (4) бесконечна, выбор $s_{1}$ произволен. Поскольку в дальнейшем при решении системы мы будем предполагать, что в исходной системе (4) начиная с некоторого $m$ все $\nu_{n}^{2}(n=m, m+1, \ldots)$ равны $\nu_{m}^{2}, s_{1}$ выберем так, чтобы в симметризованной системе

$$
\mu_{m}=\frac{s_{n-1}}{s_{n}}=\frac{s_{m-1}}{s_{m}} \quad(n=m, m+1, \ldots) .
$$

Теперь система (10) может быть переписана в виде

$$
\begin{aligned}
& \dot{\widetilde{B}}_{0}=i \nu_{0}^{2} s_{1} \widetilde{B}_{1}=i \mu_{0} \widetilde{B}_{1}, \\
& \dot{\widetilde{B}}_{1}=i \frac{1}{s_{1}}\left(\widetilde{B}_{0}+\widetilde{B}_{2}\right)=i \mu_{1}\left(\widetilde{B}_{0}+\widetilde{B}_{2}\right),
\end{aligned}
$$




$$
\begin{aligned}
\dot{\widetilde{B}}_{2} & =i \frac{s_{1}}{s_{2}}\left(\widetilde{B}_{1}+\widetilde{B}_{3}\right)=i \mu_{2}\left(\widetilde{B}_{1}+\widetilde{B}_{3}\right), \\
\ldots \ldots \ldots \ldots \ldots \ldots \ldots \ldots \ldots \ldots \ldots \ldots & \ldots \ldots \ldots \ldots \ldots \ldots \\
\dot{\widetilde{B}}_{m} & =i \frac{s_{m-1}}{s_{m}}\left(\widetilde{B}_{m-1}+\widetilde{B}_{m+1}\right)=i \mu_{m}\left(\widetilde{B}_{m-1}+\widetilde{B}_{m+1}\right),
\end{aligned}
$$

где $\mu_{k}$ определяется соотношениями

$$
\begin{aligned}
\mu_{m} & =s_{1} \frac{\nu_{1}^{2} \nu_{3}^{2} \nu_{5}^{2} \ldots \nu_{m-1}^{2}}{\nu_{2}^{2} \nu_{4}^{2} \ldots \nu_{m-2}^{2}}(m-\text { четное }), \\
\mu_{m} & =\frac{1}{s_{1}} \frac{\nu_{2}^{2} \nu_{4}^{2} \ldots \nu_{m-1}^{2}}{\nu_{1}^{2} \nu_{3}^{2} \ldots \nu_{m-2}^{2}}(m-\text { нечетное }), \\
\mu_{m+1} & =\frac{1}{s_{1}} \frac{\nu_{2}^{2} \nu_{4}^{2} \ldots \nu_{m}^{2}}{\nu_{1}^{2} \nu_{3}^{2} \ldots \nu_{m-1}^{2}}(m-\text { четное }), \\
\mu_{m+1} & =s_{1} \frac{\nu_{1}^{2} \nu_{3}^{2} \nu_{5}^{2} \ldots \nu_{m}^{2}}{\nu_{2}^{2} \nu_{4}^{2} \ldots \nu_{m-1}^{2}}(m-\text { нечетное }) .
\end{aligned}
$$

Для дальнейшего выберем $s_{1}$ так, чтобы $\mu_{m}=\mu_{m+1}$. Теперь

$$
\begin{aligned}
& s_{1}=\frac{\nu_{2}^{2} \nu_{4}^{2} \ldots \nu_{m-2}^{2}}{\nu_{1}^{2} \nu_{3}^{2} \ldots \nu_{m-1}^{2}} \nu_{m} \quad(m-\text { четное }), \\
& s_{1}=\frac{\nu_{2}^{2} \nu_{4}^{2} \ldots \nu_{m-1}^{2}}{\nu_{1}^{2} \nu_{3}^{2} \ldots \nu_{m-2}^{2}} \cdot \frac{1}{\nu_{m}}(m-\text { нечетное })
\end{aligned}
$$

и соответственно

$$
\begin{aligned}
\mu_{0} & =\frac{\nu_{0}^{2} \nu_{2}^{2} \ldots \nu_{m-2}^{2}}{\nu_{1}^{2} \nu_{3}^{2} \ldots \nu_{m-1}^{2}} \nu_{m}, \\
\mu_{1} & =\frac{\nu_{1}^{2} \nu_{3}^{2} \ldots \nu_{m-1}^{2}}{\nu_{2}^{2} \nu_{4}^{2} \ldots \nu_{m-2}^{2}} \frac{1}{\nu_{m}}(m-\text { четное }) ; \\
\mu_{0} & =\frac{\nu_{0}^{2} \nu_{2}^{2} \ldots \nu_{m-1}^{2}}{\nu_{1}^{2} \nu_{3}^{2} \ldots \nu_{m-2}^{2}} \frac{1}{\nu_{m}}, \\
\mu_{1} & =\frac{\nu_{1}^{2} \nu_{3}^{2} \ldots \nu_{m-2}^{2}}{\nu_{2}^{2} \nu_{4}^{2} \ldots \nu_{m-1}^{2}} \nu_{m} \quad(m-\text { нечетное }) ; \\
\mu_{k} & =\frac{\nu_{k}^{2} \nu_{k+2}^{2} \ldots \nu_{m-2}^{2}}{\nu_{k+1}^{2} \nu_{k+3}^{2} \ldots \nu_{m-1}^{2}} \nu_{m} \quad(m, k-\text { оба четные или нечетные }), \\
\mu_{k} & =\frac{\nu_{k}^{2} \nu_{k+2}^{2} \ldots \nu_{m-1}^{2}}{\nu_{k+1}^{2} \nu_{k+3}^{2} \ldots \nu_{m-2}^{2}} \frac{1}{\nu_{m}} \quad(m, k \text { противоположной четности }), \\
\mu_{n} & =\nu_{m}^{2}(n=m, m+1, \ldots) .
\end{aligned}
$$

Выполняя замену переменных $\tau=\nu_{m} t$, получим окончательно

$$
\frac{d B_{0}}{d \tau}=i \gamma_{0} B_{1}(\tau)
$$




$$
\begin{aligned}
\frac{d B_{1}}{d \tau} & =i \gamma_{1}\left(B_{0}(\tau)+B_{2}(\tau)\right), \\
\cdots & \ldots \ldots \ldots \ldots \ldots \ldots \ldots, \ldots \ldots, \\
\frac{d B_{m-1}}{d \tau} & =i \gamma_{m-1}\left(B_{m-2}(\tau)+B_{m}(\tau)\right), \\
\frac{d B_{n}}{d \tau} & =i\left(B_{n-1}(\tau)+B_{n+1}(\tau)\right) \quad(n=m, m+1, \ldots),
\end{aligned}
$$

при этом

$$
\begin{aligned}
\gamma_{0} & =\frac{\nu_{0}^{2} \nu_{2}^{2} \ldots \nu_{m-2}^{2}}{\nu_{1}^{2} \nu_{3}^{2} \ldots \nu_{m-1}^{2}}(m-\text { четное }) \\
\gamma_{0} & =\frac{\nu_{0}^{2} \nu_{2}^{2} \ldots \nu_{m-1}^{2}}{\nu_{1}^{2} \nu_{3}^{2} \ldots \nu_{m}^{2}}(m-\text { нечетное }) \\
\ldots \ldots \ldots \ldots \cdots \cdots \cdots & \\
\gamma_{k} & =\frac{\nu_{k}^{2} \nu_{k+2}^{2} \ldots \nu_{m-2}^{2}}{\nu_{k+1}^{2} \nu_{k+3}^{2} \cdots \nu_{m-1}^{2}}(m, k-\text { оба нечетные или оба четные }), \\
\gamma_{k} & =\frac{\nu_{k}^{2} \nu_{k+2}^{2} \ldots \nu_{m-1}^{2}}{\nu_{k+1}^{2} \nu_{k+3}^{2} \cdots \nu_{m}^{2}}(m, k \text { противоположной четности }), \\
\gamma_{m} & =\gamma_{m+1}=\cdots=1
\end{aligned}
$$

Отметим, что возможность “замораживания" коэффициентов $\left\{\nu_{i}\right\}$ начиная с некоторого номера (т.е. замена их константой) может быть реализована на основании общей теоремы, очерчивающей рамки теории (см. приложение 2). В самом деле, на основании этой теоремы выбор параметров $\left\{\nu_{i}\right\}$ вообще произволен при достаточно больших номерах $m$. Тем не менее вопросы сходимости и выбор именно этой аппроксимации для параметров $\left\{\nu_{i}^{2}\right\}$ обсуждаются ниже.

\section{4. РЕШЕНИЕ ОСНОВНОЙ СИСТЕМЫ УРАВНЕНИЙ}

В векторной форме система (16) может быть записана следующим образом:

$$
\frac{d \vec{B}(\tau)}{d \tau}=\Gamma \vec{B}(\tau)
$$

где

$$
\Gamma=\left(\begin{array}{ccccccc}
0 & i \gamma_{0} & 0 & 0 & 0 & \ldots & 0 \\
i \gamma_{1} & 0 & i \gamma_{1} & 0 & 0 & \ldots & 0 \\
0 & i \gamma_{2} & 0 & i \gamma_{2} & 0 & \ldots & 0 \\
\ldots & \ldots & \ldots & \ldots & \ldots & \ldots & \ldots
\end{array}\right), \quad \gamma_{k}>0
$$

Решение системы (18) будем искать с помощью оператора резольвенты $R_{\lambda}[15]$ :

$$
B(\tau)=\frac{1}{2 \pi i} \oint d \lambda e^{\lambda t} R_{\lambda} x_{0},
$$

где контур интегрирования охватывает весь спектр, $x_{0}=B(0)=(1,0, \ldots 0, \ldots)$ - начальное условие,

$$
R_{\lambda} x_{0}=(\lambda E-\Gamma)^{-1} x_{0}=\tilde{z} .
$$


Таким образом, для реализации соотношения (20) нам необходимо знать спектр оператора Г. Для удобства чтения приведем известные определения [16].

ОПрЕДЕЛЕНИЕ 1. Значение $\lambda$ называют собственным значением оператора $A$, если уравнение $(A-\lambda E) x=0$ при данном $\lambda$ имеет, кроме тривиального, некоторое другое ограниченное решение.

ОПрЕДЕЛЕНИЕ 2. Точка $\lambda$ называется регулярной точкой оператора $A$, если сушествует единственное ограниченное решение уравнения $(A-\lambda E) x=y$ при любом $y$.

ОПреДЕЛЕниЕ 3. Совокупность всех значений $\lambda$, не являющихся регулярными, называется спектром оператора $A$.

Отметим, что, в частности, все собственные значения оператора принадлежат спектру и называются точками дискретного спектра. Мы будем далее искать спектр оператора Г как множество нерегулярных точек этого оператора. Легко показать, что спектр матрицы Г чисто мнимый. Для этого представим матрицу в виде произведения двух матриц: $\Gamma=D C$, где $D$ - диагональная положительно-определенная матрица, а $C$ косоэрмитова $\left(C^{*}=-C\right)$ :

$$
D=\left(\begin{array}{cccccc}
\gamma_{0} & 0 & 0 & 0 & \ldots & 0 \\
0 & \gamma_{1} & 0 & 0 & \ldots & 0 \\
0 & 0 & \gamma_{2} & 0 & \ldots & 0 \\
\ldots & \ldots & \ldots & \ldots & \ldots & \ldots
\end{array}\right), \quad C=\left(\begin{array}{ccccccc}
0 & i & 0 & 0 & 0 & \ldots & 0 \\
i & 0 & i & 0 & 0 & \ldots & 0 \\
0 & i & 0 & i & 0 & \ldots & 0 \\
\ldots & \ldots & \ldots & \ldots & \ldots & \ldots & \ldots
\end{array}\right) .
$$

Теперь из (18) имеем (знак вектора далее опускаем) $\dot{B}=D C B$. Поскольку $D$ положительно-определена, из нее можно извлечь квадратный корень: $(\sqrt{D})^{-1} \dot{B}=\sqrt{D} C B$. Пусть $y=(\sqrt{D})^{-1} B$. Тогда $\dot{y}=(\sqrt{D} C \sqrt{D}) y$. Матрица $\sqrt{D} C \sqrt{D}$ косоэрмитова, следовательно, ее спектр чисто мнимый. Но спектры матриц $D C=\Gamma$ и $\sqrt{D} C \sqrt{D}$ совпадают, поскольку любая регулярная точка матрицы $D C$ будет регулярной точкой матрицы $\sqrt{D} C \sqrt{D}$ и обратно. В этом можно убедиться непосредственно либо воспользоваться критерием регулярности [16].

Покажем далее, что спектр матрицы $Г$ состоит из сегмента $[-2 i ; 2 i]$ и, быть может, дискретной части, причем дискретному спектру соответствуют значения $|\lambda|>2$.

Если точка $\lambda$ принадлежит дискретному спектру, то существует ограниченное нетривиальное решение уравнения

$$
(\lambda E-\Gamma) x=0 .
$$

Покажем, что (22) имеет ограниченное решение не при любых $\left\{\gamma_{i}\right\}$. Учитывая, что $\lambda=$ $i \omega$, перепишем $(22)$ в виде

$$
\begin{aligned}
\omega x_{0}-\gamma_{0} x_{1} & =0, \\
\omega x_{1}-\gamma_{1}\left(x_{0}+x_{2}\right) & =0, \\
\ldots \ldots \ldots \ldots \ldots \ldots \ldots \ldots & \ldots \ldots \ldots \ldots \ldots \ldots \ldots \ldots
\end{aligned}
$$

Последнее из уравнений (23) - разностное уравнение второго порядка с постоянными коэффициентами [17]. Таким образом, решение “остатка" системы начиная с номера $m$ может быть найдено в виде [17]

$$
x_{m-1}=1, \quad x_{m}=\sigma, \quad x_{n}=\sigma^{n-(m-1)},
$$


где $\sigma$-решение характеристического уравнения

$$
\begin{aligned}
& \sigma^{2}-\omega \sigma+1=0 \\
& \sigma=\frac{\omega}{2} \pm \sqrt{\frac{\omega^{2}}{4}-1}
\end{aligned}
$$

Пусть $|\omega|>2$, тогда $\sigma$ - действительное число. Поскольку $\sigma_{1} \sigma_{2}=1$, одно из решений удовлетворяет условию $\left|\sigma_{1}\right|<1$, а другое - $\left|\sigma_{2}\right|>1$, причем $\sigma+\frac{1}{\sigma}=1$. Выберем ограниченное решение $(25) \sigma_{1}=\sigma$ и найдем последовательно из $(23) x_{m-2}, x_{m-3}, \ldots x_{1}, x_{0}$ :

$$
\begin{aligned}
x_{m-2} & =\frac{\omega}{\gamma_{m-1}} x_{m-1}-x_{m}=\frac{\omega}{\gamma_{m-1}}-\sigma, \\
x_{m-3} & =\frac{\omega}{\gamma_{m-2}} x_{m-2}-x_{m-1}=\frac{\omega}{\gamma_{m-2}} x_{m-2}-1, \\
\ldots \ldots \ldots \ldots \ldots \ldots \ldots \ldots \ldots \ldots \ldots \ldots \ldots \ldots \ldots \ldots \ldots \ldots \ldots \ldots & \ldots \ldots \ldots \\
x_{0} & =\frac{\omega}{\gamma_{1}} x_{1}-x_{2} .
\end{aligned}
$$

Однако найденные таким образом $x_{1}$ и $x_{0}$ не всегда удовлетворяют первому из уравнений $(24)$, и, следовательно, дискретный спектр сушествует не всегда, но если и сушествует, то при условии $|\lambda|>2$.

Пусть теперь $|\omega| \leqslant 2$. Тогда $(25)$ - комплексное число. Поскольку $\sigma \sigma^{*}=1,|\sigma|=1$, то $\sigma=l^{i \varphi}$. Следовательно, оба решения $\sigma_{1}$ и $\sigma_{2}$ ограничены. Точки $|\lambda| \leqslant 2$ не являются регулярными точками оператора $\Gamma$, т.к. при любых $y$ сушествуют два ограниченных решения уравнения $(\lambda E-\Gamma) x=y$. Таким образом, сегмент $|\lambda| \leqslant 2$ принадлежит непрерывному спектру.

Теперь нам надо решить систему, следующую из (21):

$$
(\lambda E-\Gamma) \tilde{z}=x_{0} .
$$

Сделаем замену $\lambda=i \omega$ и сначала удовлетворим всем уравнениям (27), кроме первого, т.е. найдем вектор $z=\left(z_{0}, z_{1}, \ldots, z_{n}, \ldots\right)$, удовлетворяющий следующей системе:

$$
\begin{aligned}
\omega z_{1}-\gamma_{1}\left(z_{0}+z_{2}\right) & =0, \\
\omega z_{2}-\gamma_{2}\left(z_{1}+z_{3}\right) & =0 \\
\ldots \ldots \ldots \ldots \ldots \ldots \ldots \ldots \ldots & \ldots \ldots \ldots \\
\omega z_{m-1}-\gamma_{m-1}\left(z_{m-2}+z_{m}\right) & =0 \\
\omega z_{n}-\left(z_{n-1}+z_{n+1}\right) & =0, \quad n=m, m+1, \ldots .
\end{aligned}
$$

Начиная с $n=m$ решение можно записать в виде [17] $z_{n}=\sigma^{n-(m-1)}$, причем $z_{m-1}=1, z_{m}=\sigma, z_{m+1}=\sigma^{2}, \ldots$, где $\sigma$ удовлетворяет уравнению (25). Подставляя в (28) значения $z_{m-1}, z_{m}$, найдем последовательно $z_{m-2}, z_{m-3}, \ldots, z_{1}, z_{0}$ :

$$
\begin{aligned}
z_{m-2} & =\frac{\omega}{\gamma_{m-1}} z_{m-1}-z_{m}=\frac{\omega}{\gamma_{m-1}}-\sigma, \\
z_{m-3} & =\frac{\omega}{\gamma_{m-2}} z_{m-2}-z_{m-1}=\frac{\omega}{\gamma_{m-2}} z_{m-2}-1, \\
\ldots \ldots \ldots \ldots \ldots \ldots \ldots \ldots \ldots \ldots \ldots \ldots \ldots \ldots \ldots \ldots & \ldots \ldots \ldots \\
z_{1} & =\frac{\omega}{\gamma_{2}} z_{2}-z_{3}, \\
z_{0} & =\frac{\omega}{\gamma_{1}} z_{1}-z_{2} .
\end{aligned}
$$


Чтобы удовлетворить первому из уравнений системы $(27)$ и тем самым найти вектор $\tilde{z}$, надо $z$ поделить на $i\left(\omega z_{0}-\gamma_{0} z_{1}\right)$.

Таким образом, соотношение (20) запишется в виде

или, переходя к компонентам,

$$
B(\tau)=\frac{1}{2 \pi i} \oint l^{i \omega \tau} \tilde{z}(\omega) d(i \omega)=\frac{1}{2 \pi i} \oint l^{i \omega \tau} \frac{z d \omega}{\omega z_{0}-\gamma_{0} z_{1}}
$$

$$
B_{0}(\tau)=\frac{1}{2 \pi i} \oint l^{i \omega \tau} \frac{z_{0} d \omega}{\omega z_{0}-\gamma_{0} z_{1}} .
$$

В соответствии с теорией [18] интеграл (30) представляет собой сумму интегралов вокруг непрерывного спектра и вокруг точек дискретного спектра, если таковой имеется. Рассмотрим интеграл (30) по контуру вокруг сегмента $[-2,2]$. Функция $\tilde{z}_{0}=\frac{z_{0}}{\omega z_{0}-\gamma_{0} z_{1}}$, стояшая под знаком интеграла (30), зависит от $\omega$ и $\sigma$, связанных соотношением (25). В точках $\omega= \pm 2$ функция $\sigma$ из (25) имеет точки ветвления. Каждой точке $\omega$ соответствуют две точки $\sigma_{1}$ и $\sigma_{2}$, связанные соотношением $\sigma_{1} \sigma_{2}=1$. Контур, охватываюший обе эти точки (с разрезом по сегменту $[-2,2]$ ), не меняет знака корня, т.е. функция допускает выделение двух однозначных ветвей. Эти ветви отображают внешность отрезка $[-2,2]$ на внутренность и внешность единичной окружности. Отображение (25) обратно к отображению $\omega=\sigma+\frac{1}{\sigma}$. Это отображение преобразует верхнюю полуокружность $|\sigma|=1$ в нижний берег сегмента $[-2,2]$, а нижнюю полуокружность - в верхний берег $[-2,2]$. Таким образом, вычисление интеграла сводится к интегрированию вдоль двух берегов отрезка $[-2,2]$. Движение по нижнему берегу $(-2 \rightarrow+2)$ соответствует $\operatorname{Im} \sigma>0$, а движение по верхнему берегу $(-2 \rightarrow+2)$ соответствует $\operatorname{Im} \sigma<0$. Таким образом, интеграл (30) перепишется в виде

Покажем, что

$$
B_{0}(\tau)=\frac{1}{2 \pi i} \int_{-2}^{2} l^{i \omega \tau} \operatorname{Im} \frac{z_{0} d \omega}{\omega z_{0}-\gamma_{0} z_{1}}
$$

Отметим, что

$$
\operatorname{Im} \frac{z_{0} d \omega}{\omega z_{0}-\gamma_{0} z_{1}}=\frac{i \gamma_{0} \sqrt{1-\frac{\omega^{2}}{4}}}{\left|\omega z_{0}-\gamma_{0} z_{1}\right|^{2}}
$$

$$
\begin{aligned}
\tilde{z}_{0} & =\frac{z_{0}}{\omega z_{0}-\gamma_{0} z_{1}}=\frac{z_{0}\left(\omega z_{0}^{*}-\gamma_{0} z_{1}^{*}\right)}{\left|\omega z_{0}-\gamma_{0} z_{1}\right|^{2}}, \\
\operatorname{Im} \tilde{z}_{0} & =-\operatorname{Im} \frac{\gamma_{0} z_{0} z_{1}^{*}}{\left|\omega z_{0}-\gamma_{0} z_{1}\right|^{2}} .
\end{aligned}
$$

Поскольку знаменатель (33) - действительное число, будем следить за мнимой частью числителя. Перепишем систему (9), домножая первое уравнение на $z_{1}^{*}$, второе на $z_{2}, \ldots$ :

$$
\begin{aligned}
z_{1}^{*} \mid z_{0} & =\frac{\omega}{\gamma_{1}} z_{1}-z_{2}, \\
z_{2} \mid z_{1}^{*} & =\frac{\omega}{\gamma_{2}} z_{2}^{*}-z_{3}^{*}, \\
z_{3}^{*} \mid z_{2} & =\frac{\omega}{\gamma_{3}} z_{3}-z_{4}, \\
\ldots \ldots \ldots \ldots \ldots \ldots \ldots \ldots, & \ldots \ldots \text { четное }), \\
z_{m-1}^{*} \mid z_{m-2} & =\frac{\omega}{\gamma_{m-1}} z_{m-1}-z_{m}(m-\text { нечетное }) . \\
z_{m-1} \mid z_{m-2}^{*} & =\frac{\omega}{\gamma_{m-1}} z_{m-1}^{*}-z_{m}^{*}(m-\text { неч }
\end{aligned}
$$


Тогда $\operatorname{Im} z_{0} z_{1}^{*}=-\operatorname{Im} z_{1}^{*} z_{2}=\operatorname{Im} z_{2} z_{3}^{*}$ и т.д. Пусть $m$ - четное число. Тогда и $(m-2)$ - четное. Так как $z_{m-1=1}, z_{m}=\sigma, z_{m+1}=\sigma^{2}, \ldots$, то

$$
\begin{aligned}
\operatorname{Im} z_{0} z_{1}^{*} & =-\operatorname{Im} z_{1}^{*} z_{2}=\operatorname{Im} z_{2} z_{3}^{*}=\operatorname{Im} z_{m-2} z_{m-1}^{*}= \\
& =-\operatorname{Im} z_{m} z_{m-1}^{*}=-\operatorname{Im} \sigma=-i \sqrt{1-\frac{\omega^{2}}{4}} .
\end{aligned}
$$

Подставляя этот результат в (33), получим выражение (32). Точно так же (и с аналогичным результатом) можно рассмотреть и нечетное $m$. Покажем теперь, что для знаменателя (33) справедливо соотношение

$$
\left|\lambda z_{0}-\gamma_{0} z_{1}\right|^{2}=P_{2 m}\left(\lambda^{2}\right)
$$

где $P_{2 m}\left(\lambda^{2}\right)$ - многочлен степени $2 m$ от $\lambda^{2}$,

$$
\left|\lambda z_{0}-\gamma_{0} z_{1}\right|^{2}=\lambda z_{0} z_{0}^{*}-\gamma_{0} \lambda 2 \operatorname{Re} z_{0} z_{1}^{*}+\gamma_{0}^{2} z_{1} z_{1}^{*} .
$$

Пусть заданы $m$ констант: $\gamma_{0}, \gamma_{1}, \ldots, \gamma_{m-1}, \gamma_{m}=1$. Тогда $z_{m-1}=1, z_{m}=\sigma$. учетом этого обстоятельства получим выражение для $z_{m-k} z_{m-k}^{*}$ из (29):

$$
\begin{aligned}
P_{0}^{(m)} & =z_{m-1} z_{m-1}^{*}=1 \\
P_{2}^{(m-1)} & =z_{m-2} z_{m-2}^{*}=\frac{\lambda^{2}}{\gamma_{m-1}^{2}}-\frac{\lambda}{\gamma_{m-1}}\left(\sigma+\sigma^{*}\right)+\sigma \sigma^{*}=\frac{\lambda^{2}}{\gamma_{m-1}^{2}}-\frac{\lambda}{\gamma_{m-1}}+1, \\
z_{m-3} z_{m-3}^{*} & =\frac{\lambda^{2}}{\gamma_{m-2}^{2}} z_{m-2} z_{m-2}^{*}-\frac{\lambda}{\gamma_{m-2}}\left(z_{m-2} z_{m-1}^{*}+z_{m-2}^{*} z_{m-1}\right)+z_{m-1} z_{m-1}^{*}= \\
& =\frac{\lambda^{2}}{\gamma_{m-2}^{2}} P_{2}^{(m-1)}-\frac{\lambda}{\gamma_{m-2}} 2 \operatorname{Re}\left(z_{m-2} z_{m-1}^{*}\right)+1 .
\end{aligned}
$$

Учитывая, что

получим

$$
2 \operatorname{Re}\left(z_{m-2} z_{m-1}^{*}\right)=2 \operatorname{Re}\left(\frac{\lambda}{\gamma_{m-1}}-\sigma\right)=\lambda\left(\frac{2}{\gamma_{m-1}}-1\right),
$$

$$
P_{4}^{(m-1)}=z_{m-3} z_{m-3}^{*}=\frac{\lambda^{2}}{\gamma_{m-2}^{2}} P_{2}^{(m-1)}-\frac{\lambda}{\gamma_{m-2}}\left(\frac{2}{\gamma_{m-1}}-1\right)+1 .
$$

По индукции с помощью громоздких, хотя и очевидных, преобразований можно показать, что (36) принимает форму

$$
\begin{aligned}
P_{2 m}\left(\lambda^{2}\right)= & \left|\lambda z_{0}-\gamma_{0} z_{1}\right|^{2}=\lambda^{2} P_{2(m-1)}^{(1)}-\gamma_{0} \lambda^{2}\left[\frac{2}{\gamma_{1}} P_{2(m-2)}^{(2)}-\right. \\
& \left.-\left(\frac{2}{\gamma_{2}} P_{2(m-3)}^{(3)}-\left(\cdots-\left(\frac{2}{\gamma_{m-1}}-1\right)\right) \ldots\right)\right]+\gamma_{0}^{2} P_{2(m-2)}^{(2)} .
\end{aligned}
$$

Нам остается рассмотреть интегрирование по контуру вокруг точек дискретного спектра. В этом случае $|\omega|>2, \sigma$ - действительное число, $|\sigma|<1$ :

$$
\begin{array}{ll}
\sigma & =\frac{\omega}{2}-\sqrt{\frac{\omega^{2}}{4}-1}, \quad \text { если } \quad \omega>0, \\
\sigma & =\frac{\omega}{2}+\sqrt{\frac{\omega^{2}}{4}-1}, \quad \text { если } \quad \omega<0 .
\end{array}
$$


Преобразуем выражение для вектора $\tilde{z}_{0}$ :

$$
\tilde{z}_{0}=\frac{z_{0}(\sigma)\left(\lambda z_{0}(1 / \sigma)-\gamma_{0} z_{1}(1 / \sigma)\right)}{\left[\lambda z_{0}(\sigma)-\gamma_{0} z_{1}(\sigma)\right]\left[\lambda z_{0}(1 / \sigma)-\gamma_{0} z_{1}(1 / \sigma)\right]} .
$$

Так как $\sigma+\frac{1}{\sigma}=1$ и $\sigma \frac{1}{\sigma}=1$, то в знаменателе (38) будет тот же полином от $\lambda^{2}$, что и в формуле (36). Будем по-прежнему обозначать $z_{0}(1 / \sigma)$ и $z_{1}(1 / \sigma)$ через $z_{0}^{*}$ и $z_{1}^{*}$. Обозначим числитель $(38)$ через $F(\lambda)$, тогда

$$
F(\lambda)=z_{0}\left(\lambda z_{0}^{*}-\gamma_{0} z_{1}^{*}\right)=\lambda z_{0} z_{0}^{*}-\gamma_{0} z_{0} z_{1}^{*} .
$$

Однако по доказанному ранее имеем

$$
z_{0} z_{0}^{*}=P_{2(m-1)}^{(1)}
$$

а для $z_{0} z_{0}^{*}-$ рекуррентную формулу

$$
z_{0} z_{1}^{*}=\frac{\lambda}{\gamma_{1}} P_{2(m-2)}^{(2)}-\left[\frac{\lambda}{\gamma_{2}} P_{2(m-3)}^{(3)}-\left(\frac{\lambda}{\gamma_{3}} P_{2(m-4)}^{(4)}-\left(\cdots-\left(\frac{\lambda}{\gamma_{m-1}}-\sigma\right)\right) \cdots\right)\right],
$$

если $m$ - четное, и

$$
z_{0} z_{1}^{*}=\frac{\lambda}{\gamma_{1}} P_{2(m-2)}^{(2)}-\left[\frac{\lambda}{\gamma_{2}} P_{2(m-3)}^{(3)}-\left(\frac{\lambda}{\gamma_{3}} P_{2(m-4)}^{(4)}-\left(\cdots-\left(\frac{\lambda}{\gamma_{m-1}}-\frac{1}{\sigma}\right)\right) \cdots\right)\right],
$$

если $m$ - нечетное.

\section{5. ОБСУЖ ДЕНИЕ РЕЗУЛЬТАТОВ И СРАВНЕНИЕ С ЭКСПЕРИМЕНТОМ}

Таким образом, из рассмотрения, проведенного в разделе 4 , следует, что замораживание параметров $\left\{\nu_{j}^{2}\right\}$ в исходной системе (4) позволяет свести задачу о форме ССП (форме спектров ЯМР) к квадратурам:

$$
A_{0}(t)=\frac{\gamma_{0}}{\pi} \int_{-2}^{2} \cos \left(\lambda \nu_{m} t\right) \frac{\sqrt{1-\lambda^{2} / 4}}{P_{2 m}\left(\lambda^{2}\right)} d \lambda+\sum_{k} \cos \left(\lambda \nu_{m} t\right) \operatorname{res}\left(\widetilde{z}_{0}\left(\lambda_{k}\right)\right) .
$$

Приведем явные выражения для ССП низших приближений. При $m=1$ имеем

$$
\begin{aligned}
A_{0}^{(1)}(t)= & \frac{1}{\pi} \frac{\nu_{0}^{2}}{\nu_{1}^{2}} \int_{-2}^{2} \cos \left(\lambda \nu_{1} t\right) \frac{\sqrt{1-\lambda^{2} / 4}}{\lambda^{2}\left(1-\frac{\nu_{0}^{2}}{\nu_{1}^{2}}\right)+\left(\frac{\nu_{0}^{2}}{\nu_{1}^{2}}\right)^{2}} d \lambda+ \\
& +\frac{\nu_{0}^{2}-2 \nu_{1}^{2}}{\nu_{0}^{2}-\nu_{1}^{2}} \cos \frac{\nu_{0}^{2} t}{\sqrt{\nu_{0}^{2}-\nu_{1}^{2}}},
\end{aligned}
$$

второе слагаемое в (43) соответствует дискретному спектру и появляется только при $\gamma_{0}=\frac{\nu_{0}^{2}}{\nu_{1}^{2}}>2$. При $m=2$ имеем

$$
A_{0}^{(1)}(t)=\frac{1}{\pi} \frac{\nu_{0}^{2}}{\nu_{1}^{2}} \int_{-2}^{2} \cos \left(\lambda \nu_{2} t\right) \frac{\sqrt{1-\lambda^{2} / 4}}{P_{4}\left(\lambda^{2}\right)} d \lambda+\frac{\nu_{0}^{2}-2 \nu_{1}^{2}}{\nu_{0}^{2}-\nu_{1}^{2}} \cos \frac{\nu_{0}^{2} t}{\sqrt{\nu_{0}^{2}-\nu_{1}^{2}}},
$$


где

$$
P_{4}\left(\lambda^{2}\right)=\frac{\nu_{2}^{2}\left(\nu_{2}^{2}-\nu_{1}^{2}\right)}{\nu_{1}^{4}} \lambda^{4}+\left(1-\frac{2 \nu_{0}^{2} \nu_{2}^{2}}{\nu_{1}^{4}}+\frac{\nu_{0}^{2}}{\nu_{1}^{2}}\right) \lambda^{2}+\frac{\nu_{0}^{4}}{\nu_{1}^{4}} .
$$

Разумеется, возможность ограничиться низшими приближениями теории при описании конкретных экспериментов принципиально зависит от поведения коэффициентов $\left\{\nu_{j}\right\}$ для данного вешества, что, в свою очередь, определяется его кристаллической структурой. В работе [19] было продемонстрировано, что компонента спектра ЯМР, обусловленная спинами ячейки, для широкого класса кристаллов с большим числом спинов в ячейке (от кристаллического растянутого полиэтилена в конформации трансзигзаг до простой кубической решетки монокристалла в $\mathrm{CaF}_{2}$ ) имеет форму, близкую к прямоугольной.

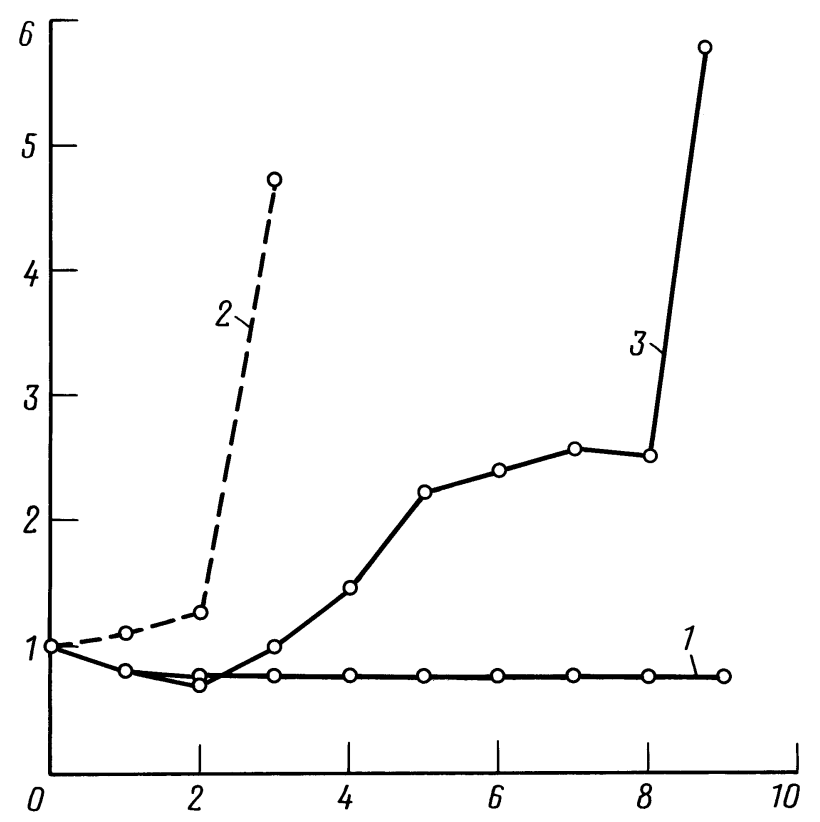

Рис. 1. Зависимость констант $\nu_{j}^{2}$ от номера $j: 1$ - прямоугольный спектр; 2 простая кубическая решетка; 3 - одномерная система.

На рис. 1 приведена зависимость $\left\{\nu_{j}^{2}\right\}$ от номера параметра $j$. Кривая 1 , соответствующая спектру прямоугольной формы, демонстрирует очень быстрый процесс замораживания констант. Численный расчет констант $\left\{\nu_{j}^{2}\right\}$ для этой модели возможен по формулам (5), (6), поскольку для прямоугольника $M_{24}=b^{2 n} / 2 n+1$, где $b$ - полуширина прямоугольного спектра. Кривая 2 соответствует $\left\{\nu_{j}^{2}\right\}$, рассчитанным по моментам полного спектра монокристалла $\mathrm{CaF}_{2}$ при ориентации внешнего магнитного поля вдоль направления [100]. Расчет возможен максимум для $j=3$, т.к. теоретические выражения для моментов трехмерных решеток имеются лиш вплоть до $M_{8}$. Отсутствие тенденции к спрямлению кривой 2 очевидным образом связано с компонентой спектра от спинов, не вошедших в ячейку. Наконец, кривая 3 соответствует спектру квазиодномерной системы ядер ${ }^{19} \mathrm{~F}$ в монокристалле фторапатита. Теоретические значения моментов для одномерной системы взяты из работы [20]. Таким образом, как следует из рис. 1 и 
результатов работы [19], на основе низших приближений теории могут быть адекватно описаны спектры широко распространенных кристаллов с плотными трехмерными и двумерными решетками, причем вклад в ССП от спинов, расположенных вне ячейки, целесообразно описывать в рамках модели $[3,14]$, используя приближение "замороженных" параметров для расчетов ССП ячейки.

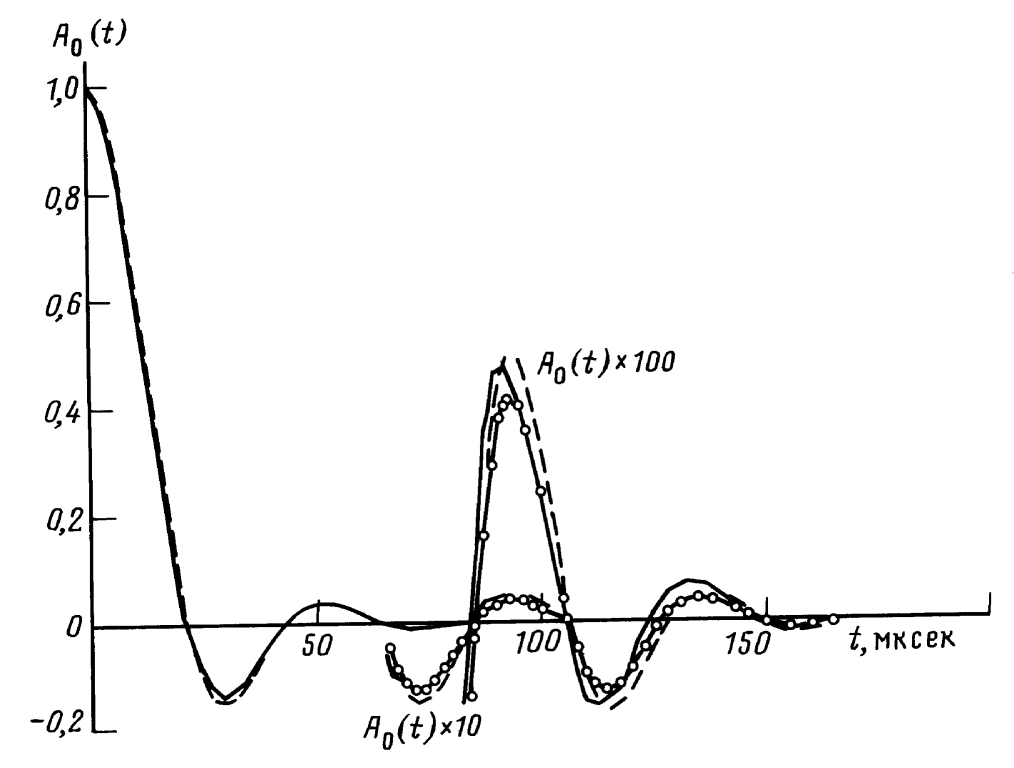

Рис. 2. ССП в монокристалле $\mathrm{CaF}_{2}$. Внешнее поле направлено вдоль направления [100]. Сплошная кривая - эксперимент, штриховая - аппроксимация с многочленом 10-го порядка, кружочки - аппроксимация с многочленом 2-го порядка.

На рис. 2 приведены теоретические и экспериментальные [21] кривые ССП для монокристалла $\mathrm{CaF}_{2}$ при ориентации внешнего поля вдоль направления [100]. Теоретические кривые были получены путем расчета по формуле (42) компоненты ССП, обусловленной ячейкой, с последующим домножением в соответствии с результатами $[3,14,21]$ на "гаусс-экспоненциальную" функцию:

$$
B(t)=\exp \left\{c\left[A-\left(A^{2}+t^{2}\right)^{1 / 2}\right]\right\} .
$$

На рис. 3 приведены результаты теории и эксперимента [22] в кристаллическом растянутом полиэтилене при ориентации поля вдоль оси молекулы. Хорошее согласие между теорией и экспериментом на рис. 2 и 3 демонстрирует адекватность сокрашенного описания ВКФ высшего порядка при помощи “замораживания" констант в кристаллах с большим числом спинов в ячейке. В работе [21], где ССП наблюдались при масштабе времен $\approx 10 T_{2}$ ( $T_{2}$ - время спин-спиновых взаимодействий), отмечено, что осциллирующая компонента ССП затухает по закону $\frac{1}{t}$. Столь большие (казалось бы!) времена должны соответствовать асимптотике, в которой в соответствии с общими физическими соображениями должна проявляться спиновая диффузия, обеспечивающая затухание $\frac{1}{t^{3 / 2}}$. Результаты расчетов, однако, показали, что выход на этот режим затухания 


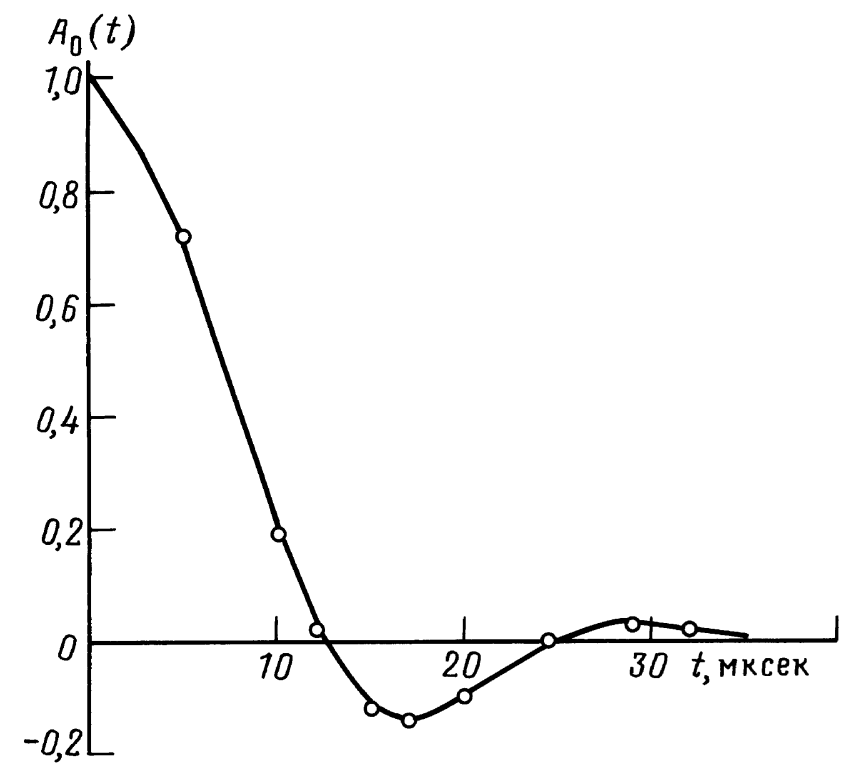

Рис. 3. ССП в монокристаллическом растянутом полиэтилене. Внешнее поле направлено вдоль оси молекулы. Точки - пробная функция, использованная в работе [22] для описания внутрикристаллического вклада (т.е. вклада ячейки $[3,14,22]$ ) в ССП. Сплошная кривая - аппроксимация с многочленом 2-го порядка.

действительно происходит, но лишь при временах $\approx 50 T_{2}$. При этом время выхода фактически не зависит от номера замораживаемой константы, т.е. время одинаково вне зависимости от порядка приближения.

\section{6. ЗАКЛЮЧЕНИЕ}

В заключение обсудим некоторые результаты работ $[23,24]$, послуживших одним из стимулов развития вышеизложенной теории. Мы уже отмечали, что в бесконечной системе уравнений (4) симметризация, вообше говоря, не однозначна. Целью изложенного способа симметризации было дальнейшее замораживание константы. В работах [23, 24] был избран иной способ симметризации, приводящий (при замораживании) к появлению двух перемежаюшихся констант и, кроме того, устанавливаюший новые искусственные связи между параметрами $\left\{\nu_{j}^{2}\right\}$. Сделанное далее без каких-либо мотивировок расцепление $[23,24]$ позволило авторам выписать два низших приближения теории. Рассчитанные ССП всего кристалла $\mathrm{CaF}_{2}$ (без выделения ячейки) неплохо совпадают с экспериментальными при не слишком больших временах. В то же время расчеты ССП для всего кристалла, выполненные нами в соответствии с вышеупомянутой теорией, и расчеты для одномерной системы привели к плохому согласию с экспериментальными результатами, что отражает принципиальные различия в сделанных приближениях с работами $[23,24]$.

Авторы благодарят П. Б. Медведева за обсуждение результатов работы. 
ПРИЛОЖКНИЕ 1

В работах $[3,14]$ показано, что компонента ССП, которая обусловлена спинами, расположенными вне ячейки, затухает со временем существенно медленнее вклада в ССП от спинов ячейки:

$$
M_{2}^{(1)} \cong 0,1 M_{2}^{(0)}
$$

$M_{2}^{(0)}$ и $M_{2}^{(1)}$ - вклады во второй момент соответственно спинов ячейки и“далеких спинов". Поэтому для формального разделения вкладов в ССП в системе уравнений (4) можно, например, воспользоваться методом возмушений в соответствии с рецептом работы [13]. Так, если предположить [13], что константа $\nu_{0}^{2}$ в первом из уравнений системы (4) является суммой двух констант, $\Delta_{0}^{2}$ и $\delta_{0}^{2}$ (сравни с формулой (П.1.1)), для "подправленного" ССП имеем [13]

$$
A_{0}^{(1)}=A_{0}^{(0)}(t)+\left(\delta_{0}^{2} / \Delta_{0}^{2}\right) \int_{0}^{1} d t_{1} \dot{A}_{0}^{(0)}\left(t_{1}\right) A_{0}^{1}\left(t-t_{1}\right) .
$$

Аналогичным образом можно выписать и дальнейшие поправки [13]. Вследствие их громоздкости и наличия выраженного малого параметра ограничимся далее обсуждением поправки низшего приближения. Уравнение (П.1.2) может быть решено с помошью преобразования Лапласа. Предполагая в соответствии с основным текстом работы, что $A_{0}^{(0)}(t)=\sin (b t) / b t$, получим

$$
\widetilde{A}_{0}^{(1)}(s)=\widetilde{A}_{0}^{(0)}(s) /\left((1-\alpha)-\alpha s \widetilde{A}_{0}^{(0)}(s)\right)=\operatorname{Arctg} \frac{b}{s} /\left((1-\alpha)-\alpha s \operatorname{Arctg} \frac{b}{s}\right),
$$

$s$-комплексная переменная, $\widetilde{A}_{0}^{(0)}(s) \widetilde{A}_{0}^{(1)}(s)$ - соответствуюшие функции времени, преобразованные по Лапласу. Спектры поглощения ЯМР, соответствующие функции, полностью аналогичной (П.1.3) для различных значений параметра $\alpha$ (здесь $\alpha=\delta_{0}^{2} / \Delta_{0}^{2}$ ), рассчитьвались нами ранее [14] в связи с иной проблемой. Однако графики работы [14] в достаточной мере иллюстрируют переход от прямоугольного спектра, соответствующего $A_{0}^{(0)}(t)$, к характерным спектрам, наблюдаемым, например, в $\mathrm{CaF}_{2}$.

Очевидно, более наглядным и физически интересным представляется разделение вкладов в ССП в системе (4), выполненное в духе популярной модели Андерсона [9]. Будем предполагать, что искомая функция $A_{0}(t)$ представима в виде произведения двух функций, одна из которых затухает к тому же сушественно медленнее другой:

$$
A_{0}(t)=C_{0}(t) B_{0}(t)
$$

Тогда в низшем приближении теории при условии $\nu_{0}^{2}=\Delta_{0}^{2}+\delta_{0}^{2}$ первое из уравнений системы (4) дает

$$
\begin{aligned}
\dot{C}_{0}(t) B_{0}(t) & =i \delta_{0}^{2} C_{0}(t) B_{1}(t), \\
\dot{B}_{0}(t) & =i \Delta_{0}^{2} B_{1}(t)
\end{aligned}
$$

что и означает разделения системы уравнений (4) на две части. Проинтегрировав первое из уравнений (П.1.5), получим

$$
C_{0}(t)=\exp \left\{i \delta_{0}^{2} \int_{0}^{t} d t_{1}\left(B_{1}\left(t_{1}\right) / B_{0}\left(t_{1}\right)\right)\right\}
$$


Учитывая, что $B_{1}(t)$ - мнимая функция времени, сделаем в (П.1.6) традиционный для модели Андерсона переход к гауссову случайному процессу:

$$
i B_{1}(t) / B_{0}(t)=-\int_{0}^{t} k(\tau) d \tau .
$$

Таким образом, получим окончательно

$$
C_{0}(t)=\exp \left\{-\delta_{0}^{2} \int_{0}^{t}(t-\tau) k(\tau) d \tau\right\}
$$

ПРИЛОЖЕНИЕ 2

ТЕОРемА. Пусть заданы система

$$
\frac{d x}{d t}=B x, \quad x=\left(x_{1}, x_{2}, \ldots, x_{n}, \ldots\right) \in l_{2} \quad[16]
$$

где $B$ - ограниченный оператор $(\|B\|=c<\infty)$, и система

$$
\frac{d y}{d t}=B_{n} y, \quad y=\left(y_{1}, y_{2}, \ldots, y_{m}, \ldots\right) \in l_{2},
$$

где $\left\{B_{n}\right\}$ - равномерно ограниченное семейство операторов $\left(\left\|B_{n}\right\|<M \forall n\right)$ такое, что $B_{n} x$ совпадает $c$ Bx в первых $n$ компонентах. Тогда на конечном интервале времени при фиксированных начальных данных $\|x-y\|_{l_{2}} \rightarrow 0$ при $n \rightarrow \infty$.

ДокАЗАТЕЛЬство. Представим оператор $B$ в виде

$$
B=B_{n}+\delta B_{n} .
$$

Так как $x \in l_{2}$, то $\left\|\delta B_{n} x\right\| \rightarrow 0 \forall x$ при $n \rightarrow \infty$. Решение системы (П.2.1) запишем следующим образом [15]:

$$
x(t)=\frac{1}{2 \pi i} \oint d \lambda e^{\lambda t} R_{\lambda}(B) x(0),
$$

где контур охватывает весь спектр оператора $B$. Для этого достаточно взять $|\lambda|>r(B)$, $r=\lim _{m \rightarrow \infty}\left(\sqrt{B^{m}}\right)^{1 / m}-$ спектральный радиус оператора $B$. Это обеспечивает сходимость ряда

$$
R_{\lambda}(B) x(0)=(\lambda I-B)^{-1} x(0)=\sum_{k=0}^{\infty} \frac{B^{k}}{\lambda^{k+1}} x(0) .
$$

Подставим (П.2.3) в (П.2.5) и запишем (П.2.5) в виде суммы двух рядов:

$$
R_{\lambda}(B) x(0)=\left[\left(\frac{1}{\lambda}+\frac{1}{\lambda^{2}} B_{n}+\frac{1}{\lambda^{3}} \cdots\right)+R_{n}\left(\lambda, B_{n}, \delta B_{n}\right)\right] x(0) .
$$

Из (П.2.6) видно, что

$$
R_{\lambda}(B) x(0)=\left(\lambda I-B_{n}\right)^{-1} x(0)+R_{n}\left(\lambda, B_{n}, \delta B_{n}\right) x(0)=R_{\lambda}\left(B_{n}\right) x(0)+R_{n} x(0) .
$$

Подставляя (П.2.7) в (П.2.4) и учитывая, что решение (П.2.2) имеет вид

получим

$$
y(t)=\frac{1}{2 \pi i} \oint d \lambda e^{\lambda t} R_{\lambda}\left(B_{n}\right) x(0),
$$

$$
x(t)-y(t)=\frac{1}{2 \pi i} \oint d \lambda e^{\lambda t} R_{n}\left(\lambda, B_{n}, \delta B_{n}\right) x(0) .
$$

Так как по условию $\left\|B_{n}\right\|<M,\left\|\delta B_{n} x(0)\right\|_{l_{2}} \rightarrow 0$, то при соответствуюшем выборе контура $\left\|R_{n}\left(\lambda, B_{n}, \delta B_{n}\right) x(0)\right\|_{l_{2}} \rightarrow 0$ при $n \rightarrow \infty$. Из-за ограниченности $\exp (\lambda t)$ на конечном интервале из (П.2.8) следует, что $\|x(t)-y(t)\|_{l_{2}} \rightarrow 0$ при $n \rightarrow \infty$, что и требовалось доказать. 


\section{Список литературы}

[1] Resibois P., De Leener M. // Phys. Rev. 1966. V. 152. P. 305; P. 318.

[2] Sauerman G., Wiegand D. // Physica. 1980. V. 103B. P. 309.

[3] Лундин А.А., Макаренко А.В. // ЖЭТФ. 1984. Т. 87. С. 999; Лундин А. А. // ЖЭТФ. 1992. T. 102. C. 352.

[4] Зобов В.Е. // ТМФ. 1990. Т. 84. №1. C. 111-119.

[5] Bohm M. et al. // Phys. Rev. B. 1994. V. 49. P. 417.

[6] Зобов В.Е., Лундин А.А. // ЖЭТФ. 1994. Т. 106. С. 1097.

[7] Bloembergen N., Purcell E. M., Pound R. V. // Phys. Rev. 1948. V. 73. P. 679.

[8] Абрагам A. Ядерный магнетизм. Гл. 4,10. М.: Мир, 1963.

[9] Anderson P. W., Weiss P. R. // Rev. Mod. Phys. 1953. V. 25. P. 269; J. Phys. Soc. (Japan). 1954. V. 9. P. 316.

[10] Kubo R., Tomita K. // J. Phys. Soc.(Japan). 1954. V. 9. P. 888.

[11] Lowe I. J., Norberg R.E. // Phys. Rev. 1957. V. 107. P. 46.

[12] Lundin A. A., Makarenko A. V., Zobov V. E. // J. Phys.: Condes. Matter. 1990. V. 2. P. 10131.

[13] Lado F., Memory J. D., Parker G.W. // Phys. Rev. B. 1971. V. 4. P. 1406.

[14] Лундин А.А., Провоторов Б. Н. // ЖЭЭТФ. 1976. Т. 70. С. 2201.

[15] Данфорд Н., Швари Д. Линейные операторы, ч.1, гл.VII. М.: ИИЛ, 1962.

[16] Люстерник Л.А., Соболев В.И. Элементы функционального анализа. М.-Л.: ГИТТЛ, 1951.

[17] Самарский A. А., Гулин А. В. Численные методы. М.: Наука, 1989.

[18] Лаврентьев М. А., Шабат Б. В. Методы теории функций комплексного переменного. М.: Наука, 1973.

[19] Лундин А. А., Макаренко А. В. // ФТТ. 1986. Т. 28. С. 2885.

[20] Sur A., Lowe I. J. // Phys. Rev. B. 1975. V. 12. P. 4597.

[21] Engelsberg M., Lowe I. J. // Phys. Rev. B. 1974. V. 10. P. 822.

[22] Федотов В.Д., Абдраиитова Н. А. // ВМС. 1980. Т. 22А. С. 624.

[23] Engelsberg M., Lowe I. J. // Phys. Rev. B. 1975. V. 12. P. 3547.

[24] Engelsberg M., Chao Nai-Cheng // Phys. Rev. B. 1975. V. 12. P. 5043.

Институт химической физики

им. Н. Н. Семенова

Поступила в редакцию 19.IV.1995 г.

V.L. Bodneva, A. A. Lundin, A. A. Milyutin

ON POSSIBILITY OF USING THE REDUCED DESCRIPTION OF THE ANISOTROPIC HEISENBERG PARAMAGNET SPIN DYNAMICS, AND THE NMR LINE-SHAPE IN SOLIDS

The problem of free induction decay calculations in solids is solved in quadratures. Existence of this solution is the consequence of the similarity relations between time correlation functions appearing in the infinite chain of the gearing differential equations. Thus, the reduced description of spin dynamics for the most spreading kinds of crystals becomes appropriate. General characteristics of the system are investigated and some theorems are proved. Theoretical results obtained for the present theory are in good agreement with experimental data. 